\title{
Three-dimensional numerical simulation of a turbulent flow around an obstacle.
}

\author{
Benahmed Lamia $^{1 *}$, Aliane Khaled ${ }^{2}$, Z. Sari Hassoun ${ }^{3}$ \\ 1, 2 Laboratory MECACOMP, Faculty of Technology, University Tlemcen, Algeria. \\ 3 Department of Mechanical Engineering, Ain Temouchent, Algeira.
}

\begin{abstract}
In this work we study the influence of the inclined shape of the lover and downstream edge of a rectangular obstacle. We analyze the dimensions of the recirculation zones, the velocity field, the kinetic energy and the pressure. A three-dimensional study was conducted using the ansys cfx calculation code. The turbulence model $\mathrm{k}-\varepsilon$ is used to model turbulence, and the governing equations are resolved by the finite volume method.
\end{abstract}

\section{NOMENCLATURE}

$\begin{array}{llc}\mathrm{Pr} & \text { Pranth Number } \\ \mathrm{U} & \text { V elocity } & (\mathrm{m} / \mathrm{s}) \\ \mathrm{u}, \mathrm{V}, \mathrm{w} & \text { next speed components }(\mathrm{x}, \mathrm{y}, \mathrm{z}) \\ \phi & \text { Physical size } & (\mathrm{Pa}) \\ \mathrm{P} & \text { Pressure } & (\mathrm{K}) \\ \mathrm{T} & \text { Temperature } & \left(\mathrm{m}^{2} / \mathrm{s}^{3}\right) \\ \varepsilon & \text { Turbulent dissipation } & (\mathrm{kg} / \mathrm{m}-\mathrm{s}) \\ \mu \mathrm{t} & \text { Eddy viscosity } & \left(\mathrm{kg} / \mathrm{m}^{3}\right) \\ \sigma_{k} & \text { Prandtl number of the turbulent } \mathrm{kinetic} \text { energy } \\ \sigma_{\varepsilon} & \text { Dissipation energy } & (\mathrm{m}) \\ \mathrm{P}_{\mathrm{k}} & \text { Rate of Production } & (\mathrm{m}) \\ \rho & \text { Air density } & (\mathrm{m} 2 / \mathrm{s} 2) \\ \mathrm{h} & \text { Canal height } & (\mathrm{m}) \\ \mathrm{H} & \text { Height of the obstacle } & \\ \mathrm{k} & \text { kinetic energy of turbulence } & \end{array}$

\section{Introduction}

The dynamics of fluids passing through stator vanes and / or turbo machine rotors is a field of study that has interested a large number of researchers. The reported work was, in general, motivated by the need to understand the complex phenomenology existing in order to improve the performance of this type of machine and to solve problems like the premature fatigue of the material related to instabilities in the flow.

*Corresponding author:benahmed_lamia91@yahoo.fr
Several experimental and numerical works have been carried out to answer the problems posed.

Hee Chang Lim et al. [1] presented a numerical simulation of flow around a surface mounted cube, placed in a turbulent boundary layer, by comparing a numerical standard model LES with experimentation. The simulations were carried out at a Reynolds number of 20000 . The numerical model used by the authors succeeded in receiving the main characteristics of the average flow and the fluctuation of the surface pressure.

In the same approach, SiniÏsa Krajnovi'c et al. [2] used the LES model of the flow around a surface-mounted cube showing that it is possible to obtain accurate results in a coarse grid simulation using a dynamic one-equation subgrid-scale model. The LDKM and OAM subgrid

models were compared. Although the two models gave similar results, there were some differences.

Yakhot et al. [3] studied Turbulent flow around a bluff body. The immersed-boundary finite-volume method was introduced to carry out a direct numerical simulation. They studied the Turbulence mean-square intensities, kinetic energy $k$, Reynolds shear stress and negative turbulence production in the region in front of the cube. They found that the emergence of negative production in front of the cube can explain the failure of some LES/RANS simulations to predict flows around a surface mounted cube. They concluded that their results support the recent trends to use a immersed-boundary method on structured grids as a tool for simulating complex turbulent flow.

Becker et al. [4] presented an experimental investigation to study the structure of the flow field around three-dimensional obstacles of different aspect ratios, in two different types of boundary layers. The dimensions of the rectangular block obstacles were chosen to represent generic shapes of buildings. The studies were performed using different kinds of flow 
visualization techniques and a Laser Doppler Anemometry (LDA) system, specially developed for wind tunnel applications and designed to provide a high spatial resolution. The results of the experiments showed the dependence of the flow structure around the obstacle on its aspect ratio, the angle of attack, the Reynolds number, and the type of boundary layer.

K. Aliane et al. [5] presented a numerical analysis of the turbulent flow inside a channel of rectangular section, with two types of obstacles in the two-dimensional case: a rectangular block obstacle and a rectangular obstacle with upstream round edge getting radius curvature 0.2 times the height of the obstacle. Several boundary conditions were explored, being the more realistic results obtained by prescribing the inlet velocity field and atmospheric pressure at the exit. The objective of this paper is to compare the special round obstacle impacts on the recirculation zone and velocity profiles considering those of the rectangular block obstacle in the twodimensional case.

The existence of the obstacle in a flow certainly causes recirculation zone located upstream and downstream of the obstacle. These zones may represent pollution areas, where the pollution remains retained.

\section{Numerical modeling}

In the problem studied, the flow considered is threedimensional, stationary and turbulent of a fluid with constant properties. In the following will be described the conservation equations including the conservation of mass, momentum and energy.

- The mass conservation equation

$$
\frac{\partial \rho}{\partial t}+\frac{\partial\left(\rho U_{i}\right)}{\partial x_{i}}=0
$$

$$
\frac{d \rho}{d t}+\rho\left(\frac{\partial u}{\partial x}+\frac{\partial v}{\partial y}+\frac{\partial w}{\partial z}\right)=0
$$

- Conservation equations of amount of movement

$$
\frac{\partial \vec{V}}{\partial t}+u \frac{\partial \vec{V}}{\partial x}+v \frac{\partial \vec{V}}{\partial y}+w \frac{\partial \vec{V}}{\partial z}=-\frac{1}{\rho} \overrightarrow{\operatorname{grad} P}+v \nabla \vec{V}
$$

Or

$$
\frac{\partial U_{i}}{\partial t}+U_{j} \frac{\partial U_{i}}{\partial x_{j}}=-\frac{1}{\rho} \frac{\partial P}{\partial x_{i}}+v \frac{\partial^{2} U_{i}}{\partial x_{j} \partial x_{j}}
$$

- $\quad$ Energy Equations

$$
\frac{\partial \bar{T}}{\partial t}+\bar{U} j \frac{\partial \bar{T}}{\partial x_{j}}=\frac{\partial}{\partial x_{j}}\left[\left(\frac{\mu}{\operatorname{Pr}}+\frac{\mu_{t}}{\operatorname{Pr}_{t}}\right) \frac{\partial \bar{T}}{\partial x_{j}}\right]+\frac{1}{\rho C_{P}} \Phi
$$

\section{Turbulence model $k-\varepsilon$}

To close the system of equations of the problem to be solved and for a much more practical approach we used the standard k$\varepsilon$ turbulence model (Launder and Spalding, 1974 [10]), this model is a semi-empirical model with 2 transport equations based on the concept of Boussinesq linking the constraints of Reynolds to average deformation rates:

For turbulent kinetic energy $\mathrm{k}$

$\frac{\partial}{\partial \mathrm{t}}(\rho \kappa)+\frac{\partial}{\partial \mathrm{x}_{\mathrm{i}}}\left(\rho \kappa \mathrm{u}_{\mathrm{i}}\right)=\frac{\partial}{\partial \mathrm{x}_{\mathrm{i}}}\left[\frac{\left(\frac{\mu+\mu_{\mathrm{t}}}{\sigma_{\kappa}}\right) \partial \kappa}{\partial \mathrm{x}_{\mathrm{i}}}\right]+\mathrm{P}_{\kappa}+\mathrm{P}_{\mathrm{b}}-\rho \varepsilon-\mathrm{Y}_{\mathrm{M}}+\mathrm{S}_{\kappa}$

Energy dissipation $\varepsilon$ :

$\frac{\partial}{\partial \mathrm{t}}(\rho \varepsilon)+\frac{\partial}{\partial \mathrm{x}_{\mathrm{i}}}\left(\rho \varepsilon \mathrm{u}_{\mathrm{i}}\right)=\frac{\partial}{\partial \mathrm{x}_{\mathrm{i}}}\left[\left(\mu+\frac{\mu_{\mathrm{t}}}{\sigma_{\varepsilon}}\right) \frac{\partial \varepsilon}{\partial \mathrm{x}_{\mathrm{i}}}\right]+\mathrm{C}_{1 \varepsilon} \frac{\varepsilon}{\kappa}\left(\mathrm{P}_{\kappa}+\mathrm{C}_{3 \varepsilon} \mathrm{P}_{\mathrm{b}}\right)-$ $\mathrm{C}_{2 \varepsilon} \rho \frac{\varepsilon^{2}}{\mathrm{k}}+\mathrm{S}_{\mathrm{\kappa}}$

$p_{k}$ Represents the term kinetic energy production

$P_{\kappa}=-\rho u_{i} u_{j} \frac{\partial u_{j}}{\partial x_{i}}$

The turbulent viscosity is calculated by:

$\mu_{\mathrm{i}}=\rho c_{\mu} \frac{\kappa^{2}}{\varepsilon}$

The empirical constants of the standard model are:

$$
C_{\mu}=0.09, C_{\varepsilon 1}=1.44, C_{\varepsilon 2}=1.92, \sigma_{k}=1.0, \sigma_{\varepsilon}=1.3
$$

\section{Geometry and boundary conditions}

The geometry of the problem considered (Fig. 1) is a threedimensional study, has three electronic components in the form of stairs, mounted in a horizontal channel length (L) and height (H).

The boundary conditions of the treaty problem are given as follows, as shown in Figure (1): 
A constant speed is given to the channel input $(U=7 \mathrm{~m} / \mathrm{s})$, the velocity is zero the lower and upper walls of the channel and above the obstacle. The transverse velocity must be zero at the entrance and at the walls

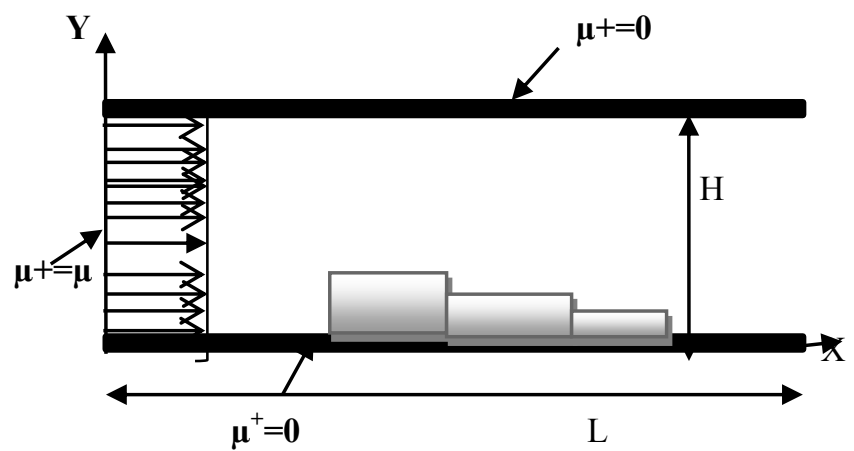

Fig 1. Geometry of computational domain

\section{The mesh}

The (Fig 2) show the grid of the mesh

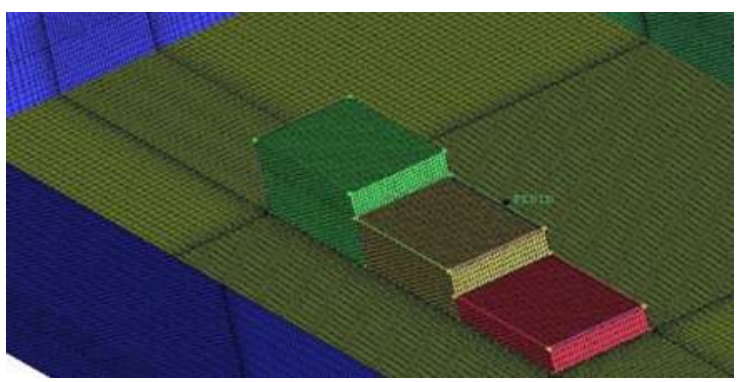

Fig 2 . Hexedric grid for configuration

\section{Results and discussion}

Various visualization techniques were used in this work. Among them are:
1. The velocity field
2. The kinetic energy
3. The pressure

\subsection{Component of the longitudinal velocity $u$}

The Fig. 3 shows shows the contours of longitudinal speed in the direction of flow where it is clear that the flow is strongly accelerated in the zone above obstacles

It is obvious that the bridge by its presence in the channel reduces the flow area causing a strong acceleration of the flow.

\footnotetext{
*Corresponding author:benahmed_lamia91@yahoo.fr
}

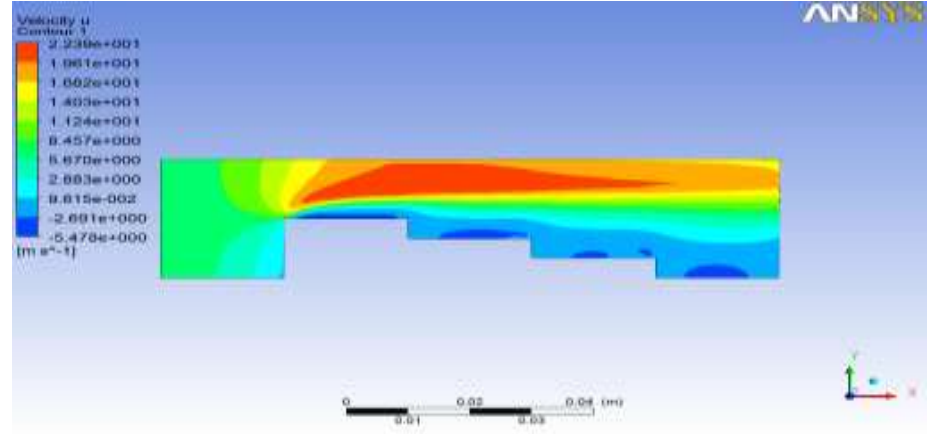

Fig 3. Contours of the the longitudinal velocity on the symmetry plane

\subsection{Component of the transversal velocity $v$}

The Fig. 4 shows the contours of the transverse in the direction of flow as the speeds reached maximum values before the first obstacle. By against downstream of each staircase can be seen very low speeds.

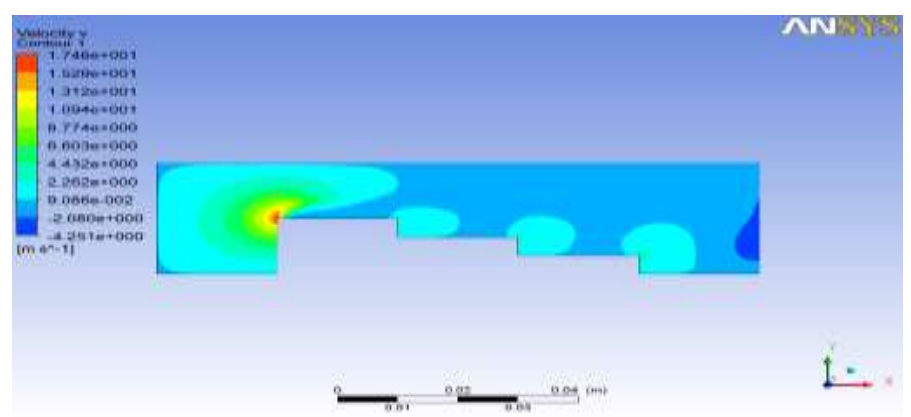

Fig 4. Contours of the transverse velocity on the symmetry plane.

\section{2 .Component of Turbulent kinetic energy}

The Fig 5 watches the contours of the kinetic energy of turbulence along the direction of flow, the high values are observed over stairs.

The rate of energy is relatively low downstream obstacles.

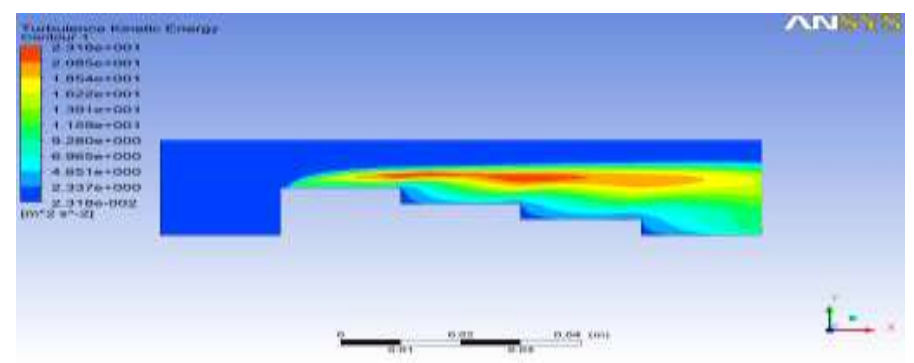

Fig 5. Turbulent kinetic energy on the symmetry plane. 


\section{The pressure field}

The Fig. 6 describes the pressure contours, a high pressure is noted upstream of the block where it reaches its maximum value. In the accelerated fluid zone discussed above (above the obstacle), a relative depression is observed (increased kinetic energy at the expense of the pressure energy).

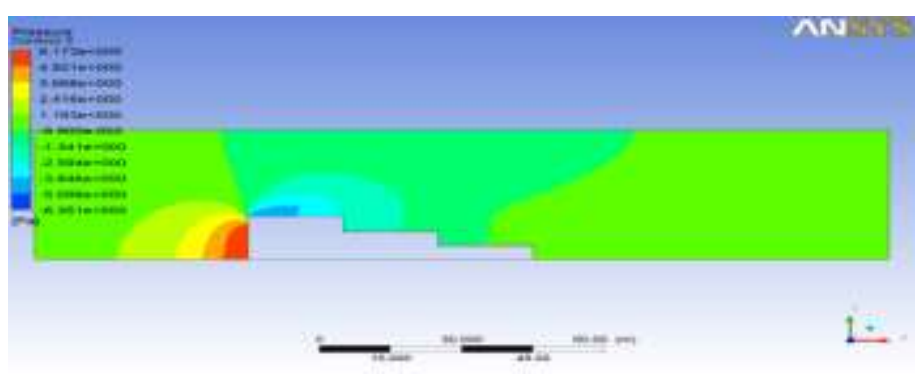

Fig 6.The pressure contours

\section{Conclusion}

This study has allowed us through a 3D simulation to analyze and understand a number of important physical aspects. In this type of flow, especially we tried to emphasize the role of the presence of obstacles in the channel, the distribution of dynamic and thermal exchanges. The analysis of simulation results confirms that:

-The presence of an obstacle in the flow leads to an increase of the dynamic exchange and thus allows the improvement of heat transfer.
- Interaction between recirculation and main flow generates a high turbulence, it is marked in the intense velocity gradients and areas of high curvature trajectories upstream of the disturbance.

\section{References}

[1] Hee Chang Lim, T.G. Thomas, Ian P. Castro, Flow around a cube in a turbulent boundary layer: LES and experiment, Journal of Wind Engineering and Industrial Aerodynamics, Volume 97, Issue 2, February 2009, Pages 96-109

[2] S. Krajnovi'c, L. Davidson, Large-eddy simulation of the flow around a bluff body, AIAA journal 40 (5), 927-936, 2002.

[3] Alexander Yakhot, Heping Liu, Nikolay Nikitin, Turbulent flow around a wall-mounted cube: A direct numerical simulation. International Journal of Heat and Fluid Flow, Volume 27, Issue 6, December 2006, Pages 994-1009.

[4] S. Becker, H. Lienhart, F. Durst, Flow around threedimensional obstacles in boundary layers, J. Wind Eng. Ind. Aerodyn., 90, 265-279, 2002.

[5] Aliane, K., Passive control of the turbulent flow over a surface mounted rectangular block obstacle and a rounded rectangular obstacle, (2011) International Review of Mechanical Engineering,(IREME), 5 (2), pp. 305-314.

*Corresponding author:benahmed lamia91@yahoo.fr 